\title{
Geographic consistency in dominant, non-typeable Haemophilus influenzae genotypes colonising four distinct Australian paediatric groups: a cohort study
}

Heidi C. Smith-Vaughan ${ }^{1,2^{*}}$, Jemima Beissbarth', Jacinta Bowman ${ }^{3}$, Kim M. Hare ${ }^{1}$, Erin P. Price ${ }^{1}$, Janessa Pickering ${ }^{4,5}$, Deborah Lehmann ${ }^{4}$, Anne B. Chang ${ }^{1,6}$, Peter S. Morris ${ }^{1,7}$, Robyn L. Marsh ${ }^{1 \dagger}$

and Amanda J. Leach ${ }^{1+}$

\begin{abstract}
Non-typeable Haemophilus influenzae (NTHi)-associated ear and respiratory diseases (including pneumonia) represent a major health burden in many parts of the world. NTHi strains retrieved from the upper airways commonly reflect those found in the lower airways. Despite growing genomic and genotyping data on NTHi, there remains a limited understanding of global and regional NTHi population structures. The aim of this study was to determine whether nasopharyngeal carriage in four Australian paediatric groups at varying risk of NTHi colonisation was dominated by the same NTHi genotypes. Genotyping data generated by PCR-ribotyping were evaluated for 3070 NTHi isolates colonising the nasopharynges of Aboriginal and non-Aboriginal children enrolled in four longitudinal studies in three separate urban and remote regions of Australia. Several NTHi PCR-ribotypes dominated in nasopharyngeal carriage, irrespective of study setting. Principal coordinates analysis confirmed a cluster of common PCR-ribotypes among all cohorts. In conclusion, we identified dominant PCR-ribotypes common to geographically disparate Australian paediatric populations. Future genomic analyses will shed further light on the precise factors underlying the dominance of certain NTHi strains in nasopharyngeal carriage.
\end{abstract}

Keywords: Non-typeable Haemophilus influenzae, Genotyping, Carriage, Paediatric, PCR-ribotyping

\section{Background}

Diseases associated with non-typeable Haemophilus influenzae (NTHi) represent a major health burden worldwide [1]. This Gram-negative bacterium is commonly associated with adult community-acquired pneumonia (CAP), particularly among those with underlying respiratory disease [2]. Because young children generally cannot expectorate, the aetiology of paediatric CAP is poorly understood and a role for NTHi remains controversial. NTHi is also associated with chronic lung diseases; for example, it is the most commonly detected

\footnotetext{
* Correspondence: heidi.smith-vaughan@menzies.edu.au

${ }^{\dagger}$ Equal contributors

${ }^{1}$ Menzies School of Health Research, Charles Darwin University, Darwin, Northern Territory, Australia

${ }^{2}$ School of Medicine, Griffith University, Gold Coast, Queensland, Australia

Full list of author information is available at the end of the article
}

pathogen in chronic obstructive pulmonary disease, a disease identified by the World Health Organization as the third leading cause of mortality globally [3]. NTHi is the most common bacterium in the lower airways of adults and children with non-cystic fibrosis bronchiectasis $[4,5]$, being cultured from the sputa of up to $70 \%$ of adults [5], and $78 \%$ of bronchoalveolar lavage specimens from Australian Indigenous children with bronchiectasis [4]. NTHi is also a major otitis media pathogen and has been detected in $89 \%$ of ear discharge specimens from children with suppurating ears in remote Australian Indigenous communities [6].

Genetic and genomic studies have shown that NTHi is a genetically diverse organism that frequently undergoes lateral gene transfer $[7,8]$, a trait that has hindered our understanding of the population biology of this organism. As an example of this diversity, the 
H. influenzae multilocus sequence typing (MLST) database (http://pubmlst.org/hinfluenzae/) currently lists 1369 NTHi isolates with MLST data from across the globe, which are represented by 966 distinct sequence types (STs). Similarly, our studies of NTHi nasopharyngeal carriage isolates have identified 130 PCR-ribotypes (PRTs) to date. Despite this high level of diversity, several studies have identified a population structure among NTHi [7,9]. Of note, MLST and whole genome analyses show some correlation between population structure and virulence potential, although no geographic clustering was detected $[7,9]$. While these data contribute to our understanding of NTHi populations, large genomic studies are necessary to improve our understanding of geographic patterns, temporal changes (including outbreaks), adaptive evolutionary pressures such as those driven by antibiotic or vaccine selection, and tissue tropism. Whole genome sequencing is ideal for exploration of bacterial population structure, and that is the direction for future studies; however, in the current study we used PRT. In addition to its cost effectiveness, PRT has the advantage of interrogating $16 \mathrm{~S}$ rDNA, which is an evolutionarily stable marker across bacterial species. This slow rate of evolution potentially provides a more robust signal for detecting strain relatedness than other genotyping methods that are based on non-ribosomal loci. We compared PRT data for 3070 nasopharyngeal carriage NTHi isolates obtained from four paediatric groups from three distinct geographic regions of Australia. Our aim was to determine whether the same NTHi PRTs dominated in nasopharyngeal carriage in these geographically distinct populations.

\section{Methods \\ Study design}

This was a retrospective cohort study that analysed NTHi genotyping data generated previously in completed studies.

\section{Participants and setting}

Study design, setting (location, dates of data collection, child eligibility criteria) are summarised in Table 1. Written informed consent was obtained from families to enrol their child in these studies. Consent forms and procedures were undertaken according to requirements of each ethical review board (see details below). Participants in Study 1 (Table 1) were primarily nonAboriginal children attending childcare in the tropical city of Darwin in the Northern Territory; these children were enrolled in a cluster randomised-controlled trial of a hygiene intervention [10]. All families were invited by letter to participate and those children whose parents consented were included in the study. Study 2 participants were Aboriginal children with acute otitis media (AOM) living in 16 remote communities across the Northern Territory; these communities ranged from those in the arid centre of Australia, up to the tropical northern "Top End" region. We attempted to screen all age-eligible children for AOM. Children with AOM whose parents consented were enrolled in a randomised-controlled trial of antibiotics

Table 1 Description of the 4 Australian paediatric non-typeable Haemophilus influenzae (NTHi)-carriage cohorts used in this study

\begin{tabular}{|c|c|c|c|c|c|c|c|}
\hline Study & $\begin{array}{l}\text { Study design, setting and } \\
\text { participant ethnicity }\end{array}$ & $\begin{array}{l}\text { Year of } \\
\text { data }\end{array}$ & $\begin{array}{l}\text { No. enrolled } \\
\text { children } \\
\text { (NTHi carriage rate) }\end{array}$ & $\begin{array}{l}\text { Child eligibility } \\
\text { age \& other } \\
\text { key } \text { criteriab }^{b}\end{array}$ & $\begin{array}{l}\text { Collection frequency } \\
\text { (No. NTHi } \\
\text { positive swabs) }\end{array}$ & $\begin{array}{l}\text { No. unique } \\
\text { NTHi typed } \\
\text { (Total NTHi typed) }\end{array}$ & $\begin{array}{l}\text { No. PRTs } \\
\text { (No. per } 100 \text { swabs) }\end{array}$ \\
\hline 1 & $\begin{array}{l}\text { Cluster RCT of a hygiene } \\
\text { intervention in } 20 \text { Darwin } \\
\text { (NT) child care centres. } \\
90 \% \text { were non-Aboriginal } \\
{[10]}\end{array}$ & 2001 & $\begin{array}{l}456 \\
(50 \%)\end{array}$ & $\begin{array}{l}0-4 \text { yrs, } \\
\text { attending } \\
3 \mathrm{~d} / \mathrm{wk}\end{array}$ & $\begin{array}{l}\text { Fortnightly for } 6 \mathrm{~m} \\
(2,012)\end{array}$ & $\begin{array}{l}2,179 \\
(2,201)\end{array}$ & $\begin{array}{l}84 \\
(4)\end{array}$ \\
\hline 2 & $\begin{array}{l}\text { RCT of azithromycin vs } \\
\text { amoxicillin for AOM in } 16 \\
\text { remote NT communities. } \\
\text { All were Aboriginal children } \\
{[11]}\end{array}$ & $2003-2005$ & $\begin{array}{l}320 \\
(316 \text { with swab data) } \\
(85 \%)\end{array}$ & $\begin{array}{l}0.5-6 \text { yrs with } \\
\text { AOM }\end{array}$ & $\begin{array}{l}\text { Days } 0 \text { and } 6-11 . \\
\text { Additionally, Day } \\
12-21 \text { if perforation. } \\
(469)\end{array}$ & $\begin{array}{l}551 \\
(569)\end{array}$ & $\begin{array}{l}73 \\
(13)\end{array}$ \\
\hline 3 & $\begin{array}{l}\text { Kalgoorlie (WA) Otitis Media } \\
\text { Research Project. } \\
\text { Prospective Aboriginal } \\
\text { cohort }[12,14]\end{array}$ & 1999-2005 & $\begin{array}{l}100 \\
(36.3 \%)\end{array}$ & $1 \mathrm{wk}$ to $24 \mathrm{~m}$ & $\begin{array}{l}\text { Ages } 1-3,6-8 \text { wks, } \\
\text { then months 4, 6, } \\
12,18,24 . \\
(193)\end{array}$ & $\begin{array}{l}231 \\
(346)\end{array}$ & $\begin{array}{l}65 \\
(28)\end{array}$ \\
\hline 4 & $\begin{array}{l}\text { Kalgoorlie (WA) Otitis Media } \\
\text { Research Project. Prospective } \\
\text { non-Aboriginal cohort [12, 14] }\end{array}$ & 1999-2005 & $\begin{array}{l}180 \\
(9.4 \%)\end{array}$ & 1 wk to $24 \mathrm{~m}$ & $\begin{array}{l}\text { Ages } 1-3,6-8 \text { wks, } \\
\text { then months } 4,6 \text {, } \\
12,18,24 \text {. } \\
(102)\end{array}$ & $\begin{array}{l}109 \\
(192)\end{array}$ & $\begin{array}{l}37 \\
(34)\end{array}$ \\
\hline
\end{tabular}


for AOM [11]. Study 3 and Study 4 included Aboriginal and non-Aboriginal children, respectively, from the semi-arid town of Kalgoorlie in southern Western Australia enrolled in a prospective study of otitis media and nasopharyngeal carriage [12]. All mothers were visited post-partum and invited to participate. None of the children in any of the four study groups (cohorts) had received the 10-valent pneumococcal $H$. influenzae protein $\mathrm{D}$ vaccine.

\section{Laboratory and statistical analyses}

For this study, we used genotyping data generated by PRT, a method that interrogates genetic polymorphisms in the $H$. influenzae ribosomal operons using restriction digestion [13]. Briefly, approximately $6 \mathrm{~kb}$ ribosomal operons (16S-5S) were amplified by long-PCR, and HaeIII restriction fragments of the resulting product were separated by agarose gel electrophoresis. PRTs were assigned based on restriction fragment lengths $(0.4$ to $1.2 \mathrm{~kb})$ following alignment to a 2-log DNA ladder (New England BioLabs, United States) and an in-house standard. To aid pattern recognition, the images were divided into 16 predetermined sections.

NTHi isolates were cultured from nasopharyngeal swabs or aspirates as described previously [4, 12]. Specimens were stored in skim milk-tryptone-glucoseglycerol broth at -70 to $-80{ }^{\circ} \mathrm{C}$ prior to culture of $10 \mu \mathrm{l}$ of specimen onto bacitracin-vancomycinclindamycin chocolate agar. NTHi were identified by colony morphology, dependence on $\mathrm{X}$ and $\mathrm{V}$ factors, and a lack of reaction with the Phadebact ${ }^{\circ}$ Haemophilus Test capsular antisera (MKL Diagnostics, Sweden). All isolates (Studies 3-4) or the majority of isolates representing all PRTs (Studies 1-2) were tested for $H$. haemolyticus misidentification by PCR targeting $h p d$ or $16 \mathrm{~S}$ rRNA genes $[14,15]$. In the Northern Territory studies, $0.2 \%(1 / 479)$ of presumptive NTHi from nasopharyngeal swabs were $H$. haemolyticus [16], while $9.5 \%$ (57/595) of presumptive NTHi from Studies 3 and 4 in Western Australia were H. haemolyticus [14]. Isolates were not tested for Protein D expression or as capsule-deficient strains of encapsulated $H$. influenzae. For Study 1 and Study 2, one NTHi colony from each positive specimen, and any that were morphologically distinct, were genotyped by PRT [13]. For Study 3 and Study 4, two colonies of presumptive NTHi where available (including any that were morphologically distinct) were subjected to PRT. A single isolate of each PRT from each swab was included in the analysis. Principal coordinates analysis of genotyping data was performed using Plymouth Routines in Multivariate Ecological Research (PRIMER)-E (PRIMER-E Ltd, UK) [17]. The similarity of PRT distributions across the four studies is shown in Fig. 1, with circles indicating each PRT and its positional location across the principal coordinates (PCO1 and PCO2) indicated on the axes; the size of the circle is proportional to the number of isolates.

\section{Results and discussion}

In the Northern Territory paediatric cohorts (Study 1 and Study 2), the mean NTHi carriage rates across all swabs were approximately $50 \%$ (unpublished) for Study 1 and $85 \%(269 / 316)$ at baseline for Study 2 [11] (Table 1). In the Kalgoorlie studies, NTHi carriage rates among the Aboriginal (Study 3) and non-Aboriginal (Study 4) children were $36.3 \%$ (183/504) and 9.4\% (98/ 1045), respectively [14]. In Study 1, 84 different PRTs were identified among 2179 NTHi isolates. In Study 2, 73 different PRTs were identified among 551 NTHi isolates. In Study 3, 65 different PRTs were detected among 231 NTHi isolates, and 37 PRTs were detected among 109 isolates in Study 4.

Although many different NTHi PRTs were present in these studies (Table 1), several NTHi PRTs dominated (Table 2), irrespective of study setting, ethnicity, or presence of AOM. In each study, the six dominant PRTs accounted for $44 \%(960 / 2179)$ (Study 1), $35 \%(193 / 551)$ (Study 2), 35 \% (81/231) (Study 3), and $46 \%$ (50/109) (Study 4) of all isolates. PRTs 3 and 8 were the most common, featuring in all four populations and being represented by $5-11 \%$ of all isolates. PRTs $15,14,13$, and 4 were the next most common PRTs, each featuring in three of the four populations (Table 2). MLST analysis (http:// pubmlst.org/hinfluenzae/) of at least one representative isolate of the six dominant PRTs in Study 1 and 2 found that all sequence types were described from the United States and Europe, with the exception of isolates representing PRTs 27, 34, and one isolate of PRT 8.

Principal coordinates analysis (PCoA) was used to visualise the PRT distribution among the four study cohorts. This analysis showed a dominant cluster of PRTs common to all studies (Fig. 1), thereby supporting the absence of geographic or population-specific clustering of NTHi PRTs. The highest number of PRTs was detected in Study 1. Higher dispersion of PRTs across PCO1 and PCO2 for Studies 1-2 (compared to Studies 3-4) reflects the increased number of PRTs unique to Studies 1-2. For Study 1, this finding likely represents the large sample (2179 NTHi isolates). However, for Study 2, this finding likely reflects the larger number of strains dominating in this population, which may result from the high NTHi carriage rates $(85 \%)$ in this cohort or its broader geographic sampling (16 remote communities). This finding could also reflect a difference in disease state (all children had a diagnosis of AOM), though we previously showed that NTHi from paired nasopharyngeal and ear discharge swabs from children with 

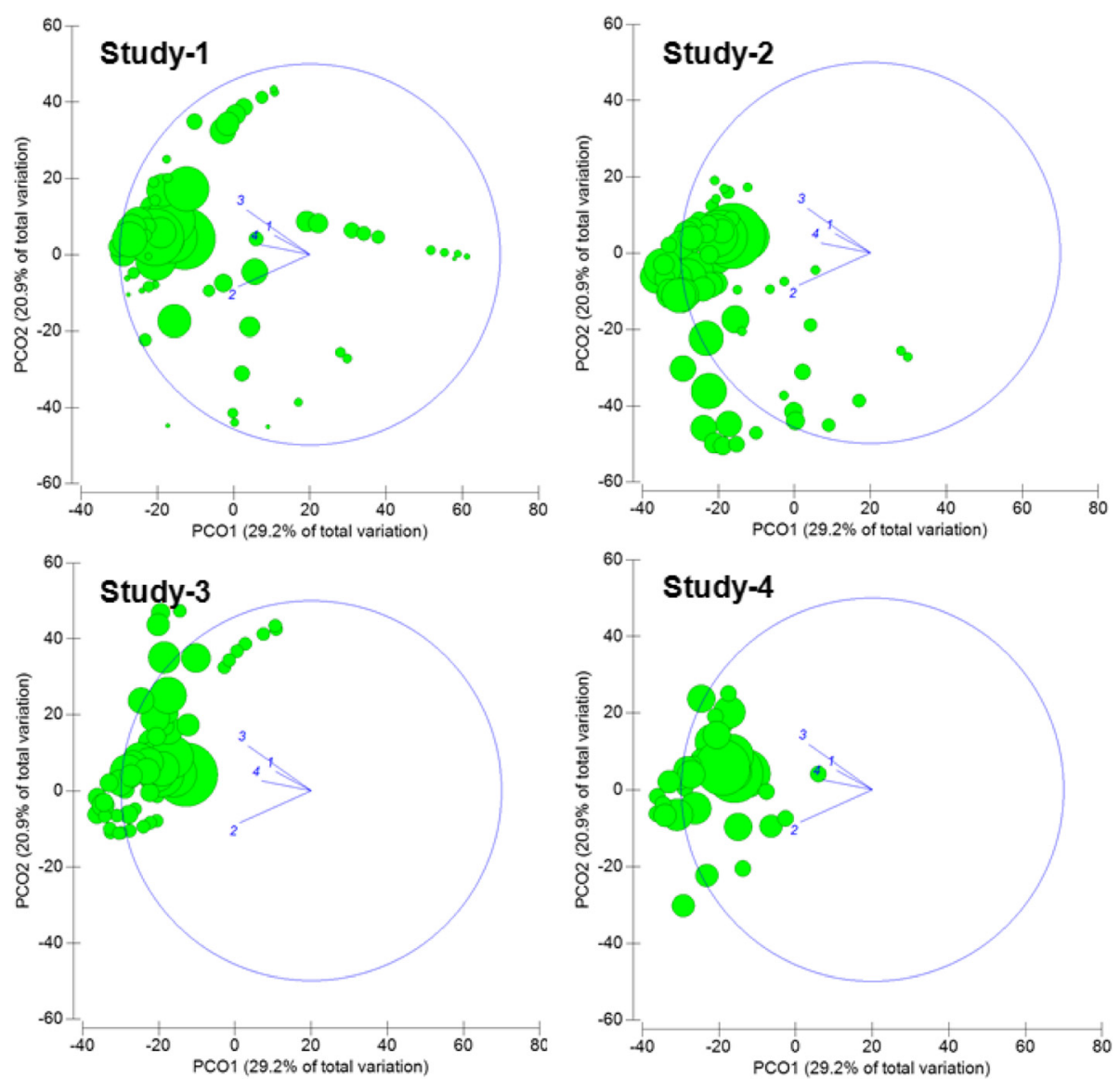

Fig. 1 Principal coordinates analysis (PCOA) visualising the similarity of PCR-ribotype (PRT) distributions between the 4 studies. Each circle indicates a PRT; the size of the circle is proportional to the number of isolates. Plots show the PRT data cloud for the 4 studies. Each figure was derived from the same PCOA and thus positional differences between the data clouds indicate differences in PRT distribution. Bubbles occurring in the same location across PCO1 (X-axis) and PCO2 (Y-axis) indicate PRTs that were common to the different studies. The vector plot shows directional effects of the PRT distribution from each study

Table 2 The 6 dominant non-typeable Haemophilus influenzae PCR-ribotypes (PRTs) in 4 populations of Australian children

\begin{tabular}{|llllr} 
Order of & $\begin{array}{l}\text { Study 1 } \\
\text { Darwin (NT) } \\
\text { primarily } \\
\text { frequency }\end{array}$ & $\begin{array}{l}\text { Study 2 } \\
\text { NT remote } \\
\text { Aboriginal } \\
\text { Aboriginal } \\
\text { PRT (\%) }\end{array}$ & $\begin{array}{l}\text { Study 3 } \\
\text { Kalgoorlie } \\
\text { Aboriginal }\end{array}$ & $\begin{array}{l}\text { Study 4 } \\
\text { Kalgoorlie } \\
\text { non- } \\
\text { Aboriginal }\end{array}$ \\
\hline 1 & PRT (\%) & PRT (\%) & PRT (\%) \\
2 & $3(10 \%)$ & $8(9 \%)$ & $3(11 \%)$ & $4(12 \%)$ \\
3 & $15(9 \%)$ & $4(8 \%)$ & $8(6 \%)$ & $20(11 \%)$ \\
4 & $8(7 \%)$ & $13(6 \%)$ & $13(5 \%)$ & $3(8 \%)$ \\
5 & $14(6.5 \%)$ & $3(5 \%)$ & $14(5 \%)$ & $14(6 \%)$ \\
6 & $13(6 \%)$ & $15(4 \%)$ & $4(4 \%)$ & $8(5 \%)$ \\
\hline
\end{tabular}

Note: Yellow $=$ PRT3; Green $=$ PRT $;$ Mustard $=$ PRT14; Purple $=$ PRT4; Dark blue $=$ PRT13; Light blue $=$ PRT15

NT, Northern Territory 


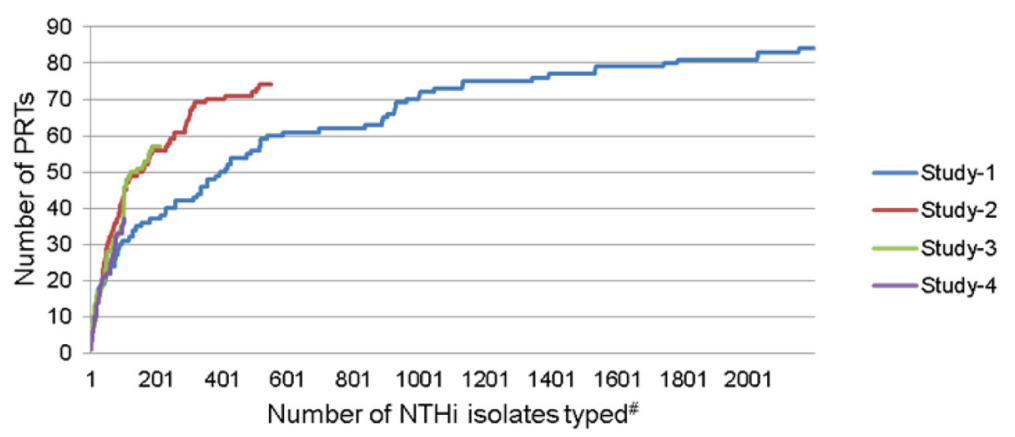

Fig. 2 Rarefaction plot demonstrating the cumulative number of new non-typeable Haemophilus influenzae (NTHi) PRTs identified versus number of NTHi isolates typed for each study. PRT, PCR-ribotype. \#Only unique isolates from each swab included

AOM with perforation were generally the same PRT (unpublished observations).

Analysis of these four diverse cohorts provided an opportunity to assess our sampling strategy for efficient surveillance of NTHi diversity within a population. Figure 2 shows the cumulative number of novel PRTs identified in each cohort against the number of NTHi isolates genotyped, irrespective of study duration. Studies 2, 3, and 4 followed a similar trajectory, in which 13-34 PRTs per 100 unique isolates were identified (Table 1). Study 2 was a 3-year study of 320 Aboriginal children up to 6 years of age, with NTHi carriage rates of up to $85 \%$ at baseline; each child was swabbed up to three times over several weeks. Study 3 was a 6-year study of 100 Aboriginal children up to 2 years of age with a $36.3 \%$ NTHi carriage rate; each child was swabbed up to 7 times over a 2 -year period. Study 4 was the non-Aboriginal cohort of 180 children from Study 3 with a $9.4 \%$ NTHi carriage rate. In contrast, Study 1 identified 4 PRTs per 100 isolates during a 6month study of 456 non-Aboriginal children up to 4 years of age with an approximately $50 \%$ NTHi carriage rate; each child was swabbed fortnightly. We have shown that low numbers of swabs with relatively low NTHi carriage (Study 3) collected less frequently and over a longer period can provide comparable information to intense, shorter studies such as Study 1.

Another carriage study design question relates to the number of isolates selected for analysis from each swab. Multiple NTHi genotypes during carriage have previously been observed and modelled [18]; however, the value of assessing multiple strain carriage in different populations is not well understood. In Studies 1-2 where additional colonies were selected only if morphologically distinct, multiple PRT carriage was detected in 8 and $17 \%$ of swabs, respectively. For Studies 3-4 where 2 colonies were selected as per protocol, including any that were morphologically distinct, multiple PRT carriage was detected in 20 and $7 \%$ of swabs, respectively. Although our data cannot inform the most appropriate number of colonies to select per specimen for measuring multiple PRT carriage, nor the method of selection, it is likely that these methods would vary by population. Indeed, the value of typing additional colonies appears to be greater for populations with high carriage rates.

In conclusion, analysis of NTHi nasopharyngeal carriage PRTs in four cohorts with varying NTHi colonisation rates allowed us to identify dominant genotypes common to four Australian paediatric populations. More than 3000 isolates from three distinct urban and remote geographic regions in Australia (Kalgoorlie in southern Western Australia, the urban Darwin region of the Northern Territory, and several remote communities across the Northern Territory) were examined. This finding provides an opportunity to study the factors conferring an advantage to the dominant PRTs in NTHi nasopharyngeal carriage, thereby advancing our understanding of the constraints that support NTHi population structure. Continued efforts to understand NTHi population dynamics will improve the identification of useful vaccine candidates for targeting universal or dominant strains, and may inform models of the potential efficacy of NTHi vaccines in current and future production pipelines. Further characterisation of carriage- and disease-related (acute [e.g. pneumonia] and chronic [e.g. bronchiectasis]) NTHi populations by whole genome sequencing or other genotyping means is necessary to determine the relevance of carriage versus disease genotype frequency, and may shed light on the factors supporting dominance of certain NTHi genotypes. Although the Australian experience may not necessarily apply elsewhere, whole genome sequence data from Australian and international NTHi isolates have (to date) demonstrated a level of core genome similarity between global and Australian NTHi populations [8]. Thus, we expect our findings to be applicable to other study settings.

\section{Acknowledgements}

We thank the families who participated in these studies for making this research possible. We also thank the many coordinators, nurses and microbiologists who contributed to the original studies, which provided the specimens used in this particular study. 


\section{Funding}

The original studies or specimen analyses were funded through the Australian National Health and Medical Research Council (NHMRC grant numbers 212044, 436023, 100009, 1023781), and Studies 3-4 through two Healthway grants (grant numbers 6028 and 10564). The Darwin component of the paper is associated with an NHMRC Centre for Research Excellence in Otitis Media of Aboriginal and Torres Strait Islander Children (grant number 1078557) and an NHMRC Centre for Research Excellence in Lung Health of Aboriginal and Torres Strait Islander Children (grant number 1040830). RM is supported by NHMRC Frank Fenner Early Career Fellowship 1034703. DL was funded through NHMRC program (grant number 353514). AC is supported by NHMRC Practitioner Fellowship 545216. AL is supported by NHMRC Elizabeth Blackburn Research Fellowship 1020561. KH is supported by NHMRC Early Career Fellowship 1072870. The funders had no role in study design, collection and analysis of data, decision to publish, or writing of the manuscript.

\section{Availability of data and materials}

Data and materials that are proprietary to Telethon Kids Institute and Menzies School of Health Research under the custodianship of the study's principal investigator can be requested from the Custodian or relevant Custodial Committees; and is subject to permission from the relevant Human Research Ethics Committees and existing contractual and third party agreements.

\section{Authors' contributions}

All the authors met ICMJE authorship criteria. JB, JP, DL, KH, PM, EP, AC, AL contributed to data acquisition. HS-V, JB, RM conducted the data analysis and interpretation. HS-V wrote the first draft of the manuscript; JB, JP, DL, KH, PM, $A L, E P, A C$ critically reviewed the manuscript. All authors advised on intellectual content of the manuscript. All authors agree with the manuscript's results and conclusions. All authors approved the final version of the manuscript.

\section{Competing interests}

The authors declare that they have no competing interests.

\section{Consent for publication}

\section{Not applicable.}

\section{Ethics approval and consent to participate}

Ethical approvals for Studies 1 (\#99/68) and $2(\# 02-44)$ were obtained from the Human Research Ethics Committees of the Menzies School of Health Research and the Northern Territory Department of Health, and from the Central Australian Human Research Ethics Committee. Study 2 was registered with the Australian Clinical Trials Registry (ACTRN 12609000691246). Ethical approval to conduct Studies 3 and 4 was provided by the Western Australian Aboriginal Health Information and Ethics Committee (WAAHIEC), the Northern Goldfields Health Service and Nursing Education Ethics Committee in Kalgoorlie, Princess Margaret Hospital Ethics Committee, and the Confidentiality of Health Information Committee of the Health Department of Western Australia. Written informed consent was obtained from families to enrol their child in these studies.

\section{Author details}

Menzies School of Health Research, Charles Darwin University, Darwin, Northern Territory, Australia. 'School of Medicine, Griffith University, Gold Coast, Queensland, Australia. ${ }^{3}$ Department of Microbiology and Infectious Diseases, PathWest Laboratory Medicine, Perth, Western Australia, Australia. ${ }^{4}$ Telethon Kids Institute, The University of Western Australia, Perth, Western Australia, Australia. ${ }^{5}$ School of Paediatrics and Child Health, University of Western Australia, Perth, Western Australia, Australia. ${ }^{6}$ Department of Respiratory and Sleep Medicine, Queensland Children's Medical Research Institute, Children's Health, Queensland University of Technology, Brisbane, Queensland, Australia. ${ }^{7}$ Royal Darwin Hospital, Darwin, Northern Territory, Australia.

Received: 14 December 2015 Accepted: 8 August 2016

\section{Published online: 18 August 2016}

\section{References}

1. Van Eldere J, Slack MP, Ladhani S, Cripps AW. Non-typeable Haemophilus influenzae, an under-recognised pathogen. Lancet Infect Dis. 2014;14:128192. PMID:25012226, http://dx.doi.org/10.1016/S1473-3099(14)70734-0.
2. Slack MP. A review of the role of Haemophilus influenzae in communityacquired pneumonia. Pneumonia. 2015;29:26-43. http://dx.doi.org/10.15172/ pneu.2015.6/520.

3. WHO. The Top 10 Causes of Death. 2014. Available from: http://www.who. int/mediacentre/factsheets/fs310/en/. Accessed 5 Jan 2015.

4. Hare KM, Grimwood K, Leach AJ, Smith-Vaughan H, Torzillo PJ, Morris PS, et al. Respiratory bacterial pathogens in the nasopharynx and lower airways of Australian indigenous children with bronchiectasis. J Pediatr. 2010;157: 1001-5. PMID:20656297, http://dx.doi.org/10.1016/j.jpeds.2010.06.002.

5. King PT, Holdsworth SR, Freezer NJ, Villanueva E, Holmes PW. Microbiologic follow-up study in adult bronchiectasis. Respir Med. 2007;101:1633-8. PMID: 17467966, http://dx.doi.org/10.1016/j.rmed.2007.03.009.

6. Smith-Vaughan HC, Binks MJ, Marsh RL, Kaestli M, Ward L, Hare KM, et al. Dominance of Haemophilus influenzae in ear discharge from Indigenous Australian children with acute otitis media with tympanic membrane perforation. BMC Ear Nose Throat Disord. 2013;13:12-3. PMID:24099576, http://dx.doi.org/10.1186/1472-6815-13-12.

7. De Chiara M, Hood D, Muzzi A, Pickard DJ, Perkins T, Pizza M, et al. Genome sequencing of disease and carriage isolates of nontypeable Haemophilus influenzae identifies discrete population structure. Proc Natl Acad Sci USA. 2014;111:5439-44. PMID:24706866, http://dx.doi.org/10.1073/pnas.1403353111.

8. Price EP, Sarovich DS, Nosworthy E, Beissbarth J, Marsh RL, Pickering J, et al. Haemophilus influenzae: using comparative genomics to accurately identify a highly recombinogenic human pathogen. BMC Genomics. 2015;16:641. PMID:26311542, http://dx.doi.org/10.1186/s12864-015-1857-x.

9. LaCross NC, Marrs CF, Gilsdorf JR. Population structure in nontypeable Haemophilus influenzae. Infect Genet Evol. 2013;14:125-36. PMID:23266487 http://dx.doi.org/10.1016/j.meegid.2012.11.023.

10. Bailie R, Morris P, Leach A, Wilson C, Dunne K, Si D, et al. The effect of additional training in recommended hygiene practices on rates of bacterial cross-infection and respiratory illness in Australian child care centres: A randomised controlled trial. Epidemiology. 2003;14:S15. http://dx.doi.org/10. 1097/00001648-200309001-00009.

11. Morris PS, Gadil G, McCallum GB, Wilson CA, Smith-Vaughan HC, Torzillo P, et al. Single-dose azithromycin versus seven days of amoxycillin in the treatment of acute otitis media in Aboriginal children (AATAAC): a double blind, randomised controlled trial. Med J Aust. 2010;192:24-9. PMID:20047544.

12. Watson K, Carville K, Bowman J, Jacoby P, Riley TV, Leach AJ, et al. Kalgoorlie Otitis Media Research Project Team. Upper respiratory tract bacterial carriage in Aboriginal and non-Aboriginal children in a semi-arid area of Western Australia. Pediatr Infect Dis J. 2006;25:782-90. PMID: 16940834, http://dx.doi.org/10.1097/01.inf.0000232705.49634.68.

13. Smith-Vaughan HC, Sriprakash KS, Mathews JD, Kemp DJ. Long PCRribotyping of nontypeable Haemophilus influenzae. J Clin Microbiol. 1995;33: 1192-5. PMID:7542264.

14. Pickering J, Smith-Vaughan H, Beissbarth J, Bowman JM, Wiertsema S, Riley TV, et al. Diversity of nontypeable Haemophilus influenzae strains colonizing Australian Aboriginal and non-Aboriginal children. J Clin Microbiol. 2014;52: 1352-7. PMID:24501028, http://dx.doi.org/10.1128/JCM.03448-13.

15. Binks MJ, Temple B, Kirkham LA, Wiertsema SP, Dunne EM, Richmond PC, et al. Molecular surveillance of true nontypeable Haemophilus influenzae: an evaluation of PCR screening assays. PLOS ONE. 2012;7:e34083. PMID: 22470516, http://dx.doi.org/10.1371/journal.pone.0034083.

16. Beissbarth J, Binks M, Scarff K, Marsh R, Nosworthy E, Chang AB, Leach AJ, Smith-Vaughan HC. Nontypeable Haemophilus influenzae and $H$. haemolyticus identification in the Northern Territory of Australia. Presented at: OMOZ, 2014; Melbourne, Australia.

17. Clarke KG, R. PRIMER v6: User Manual/Tutorial. Plymouth: PRIMER E2006

18. Smith-Vaughan HC, MCBroom J, Mathews JD. Modelling of endemic carriage of Haemophilus influenzae in Aboriginal infants in Northern Australia. FEMS Immunol Med Microbiol. 2001;31:137-43. PMID:11549421, http://dx.doi.org/10.1111/j.1574-695X.2001.tb00510.x. 